\title{
Baeyer-Villiger 单加氧酶的蛋白质改造及其催化氧化反应研究新进展
}

\author{
郑禾 $a, b$ 周玉珂 ${ }^{a}$ 林贤福 ${ }^{a}$ 吴 起*,a \\ ( ${ }^{a}$ 浙江大学化学系 杭州 310027) \\ $\left({ }^{b}\right.$ 中国科学院上海生命科学研究院 上海 200031)
}

\begin{abstract}
摘要 Baeyer-Villiger 单加氧酶(BVMO)作为一种重要的生物催化剂, 可以催化各种有机酮醛化合物的 Baeyer-Villiger 氧化反应, 以及一些含硫、硒、䂰等杂原子底物的氧化反应. Baeyer-Villiger 单加氧酶催化的氧化反应具有选择性高、 反应条件温和、高效等优点，已成为不可或缺的有机合成工具，被广泛应用于各种手性化合物的合成中. 近年来利用生 物信息学分析和基因挖掘技术，从众多微生物中找到了多种新型的 BVMO; 另外利用蛋白质工程技术对已知的野生型 BVMO 进行改造, 从而扩大底物范围, 提高热稳定性和反应活性, 改善酶的立体、区域和化学选择性. 这些都进一步扩 大了 Baeyer-Villiger 单加氧酶催化氧化反应的应用范围. 以不同底物结构的 Baeyer Villiger 氧化反应为主线, 综述了近 5 年来国内外对野生型以及蛋白质工程改造的 BVMO 催化氧化反应研究的新进展.
\end{abstract}

关键词 Baeyer-Villiger 单加氧酶; Baeyer-Villiger 氧化反应; 生物催化; 手性合成; 蛋白质改造

\section{Recent Developments in Protein Engineering and Catalytic Oxidations of Baeyer-Villiger Monooxygenase}

\author{
Zheng, He Zhou, Yuke $^{a} \quad$ Lin, Xianfu ${ }^{a} \quad$ Wu, Qi ${ }^{*}, a$ \\ ( ${ }^{a}$ Department of Chemistry, Zhejiang University, Hangzhou 310027) \\ ( ${ }^{b}$ Shanghai Institute for Biological Sciences, Chinese Academy of Sciences, Shanghai 200031)
}

\begin{abstract}
Baeyer-Villiger monooxygenase (BVMO) is an important biocatalyst for Baeyer-Villiger oxidation of various organic ketone/aldehyde compounds, and sulfur, selenium, or boron-containing heteroatoms compounds. As an indispensable tool, BVMO-catalyzed oxidation displays some advantages, such as high selectivity, mild reaction conditions and high efficiency, leading to wide applications into the synthesis of chiral compounds. In recent years, bioinformatics analysis and genome mining have been used to find more novel BVMOs from microorganisms. Besides natural substrates, these BVMOs can accept various organic compounds showing wide substrate scope. Meanwhile, protein engineering has been widely used to improve the catalytic performance of BVMOs, such as the expanded substrate scope, high thermostability and activity, high stereo-, regio- and chemo-selectivities. Based on the Baeyer-Villiger oxidation reaction with different substrate structures, the recent advancements in the research on the catalytic oxidation of wild type and protein-engineered BVMOs in the past five years are summarized.
\end{abstract}

Keywords Baeyer-Villiger monooxygenase; Baeyer-Villiger oxidation; biocatalysis; asymmetric synthesis; protein engineering

酶催化合成手性化合物是当前合成化学领域的研 究热点之一. 其中 Baeyer-Villiger 单加氧酶(Baeyer-Villiger monooxygenase, BVMO)是一种重要的生物催化剂, 可以催化各种有机酮/醛化合物的 Baeyer-Villiger (BV) 氧化反应, 以及一些含硫、硒、硼等杂原子底物的氧化 反应. BVMO 拥有广泛的多样性, 根据其黄素辅因子性
质不同主要分为 Type I 型和 II 型 BVMO. 其中 Type I 型属于 B 家族黄素单加氧酶, 依赖黄素腺嘌呤二核苷酸 (FAD) 作为辅因子和 NAPDH 作为还原剂, 目前重组可 用的 BVMO 大多属于这一类; Type II 型属于 $\mathrm{C}$ 家族黄 素单加氧酶, 依赖 NADP 和黄素单核苷酸 $(F M N)^{[1]}$. BVMO 不但拥有优异的立体选择性和区域选择性，还

* Corresponding author. E-mail: 1lc123@zju.edu.cn

Received October 19, 2018; revised December 13, 2018; published online December 28, 2018.

Project supported by the National Natural Science Foundation of China (Nos. 21472169, 21574113).

国家自然科学基金(Nos. 21472169, 21574113)资助项目. 
具有反应条件温和、高效、对环境友好等生物催化剂的 共同优点. 因此 BVMO 无可争议地成为绿色不对称 Baeyer-Villiger 氧化反应的最佳催化剂, 广泛用于手性 氧化产物的合成 ${ }^{[2]}$. BVMO 主要参与生物体内的次级代 谢, 说明在无细胞条件下也能进行催化, 有工业应用的 潜质. 随着生物信息技术和基因挖掘技术的发展，来自 微生物基因组的众多新型 BVMO 被发现和表征, 极大
地丰富了 BVMO 酶的种类和应用范围; 同时，随着蛋白 质工程技术的不断进步，利用理性设计、定向进化等方 法将已知的野生型 BVMO (表 1)改造成特定催化属性的 突变型 $\mathrm{BVMO}$, 也是目前较为热门的研究方向, 有力地 推动了 BVMO 研究和合成应用的发展 ${ }^{[3]}$. 以不同底物结 构的 Baeyer-Villiger 氧化反应为主线, 综述了近 5 年来 国内外对野生型以及蛋白质工程改造的 BVMO 催化氧

表 1 BVMO 以及其来源

Table 1 Name of BVMOs and sources

\begin{tabular}{|c|c|c|c|}
\hline Entry & BVMO 名称 & 来源 & 中文名 \\
\hline 1 & $\mathrm{BVMO}_{\text {Lepto }}$ & Leptospira biflex & \\
\hline 2 & $\mathrm{CPMO}_{\mathrm{Coma}}$ & Comamonas testosterone & 环戊酮单加氧酶 \\
\hline 3 & $\mathrm{BVMO}_{\mathrm{Cm}}$ & Cyanidioschyzon merolae & \\
\hline 4 & $\mathrm{BVMO}_{\mathrm{Pp}}$ & Physcomitrella patens & \\
\hline 5 & hFMO5 & Human & \\
\hline 6 & PAMO & Thermobifida fusca & 苯丙酮单加氧酶 \\
\hline 7 & HAPMO & Pseudomonas fluorescens $A C B$ & 4-差基苯乙酮单加氧酶 \\
\hline 8 & BVMO9 & Rhodococcus jostii RHAl & \\
\hline 9 & BVMO15 & Rhodococcus jostii RHAl & \\
\hline 10 & STMO & Rhodococcus rhodochrous & 类固醇单加氧酶 \\
\hline 11 & $\mathrm{BVMO}_{\mathrm{AFL} 456}$ & Aspergillus flavu NRRL3357 & \\
\hline 12 & $\mathrm{BVMO}_{\mathrm{AFL} 838}$ & Aspergillus flavu NRRL3357 & \\
\hline 13 & $\mathrm{BVMO}_{\mathrm{AFL} 210}$ & Aspergillus flavu NRRL3357 & \\
\hline 14 & $\mathrm{BVMO}_{\mathrm{AFL} 619}$ & Aspergillus flavu NRRL3357 & \\
\hline 15 & BVMO4 & Dietzia sp. D5 & \\
\hline 16 & $\mathrm{CHMO}_{\mathrm{JS} 666}$ & Polaromonas sp. strain JS666 & 环己酮单加氧酶 \\
\hline 17 & CPDMO & Pseudomonas sp. HI-70 & 环十五酮单加氧酶 \\
\hline 18 & CAMO & Cylindrocarpon radicicola ATCC 11011 & 环烷酮单加氧酶 \\
\hline 19 & OTEMO & Pseudomonas putida NCIMB 10007 & \\
\hline 20 & $\mathrm{CHMO}_{\text {Arthro }}$ & Arthrobacter sp & 环己酮单加氧酶 \\
\hline 21 & $\mathrm{CHMO}_{\text {Acineto }}$ & Acinetobacter $s p$ & 环己酮单加氧酶 \\
\hline 22 & $\mathrm{CHMO}_{\text {Phil }}$ & Rhodococcus sp. Phil & 环己酮单加氧酶 \\
\hline 23 & BVMO-P1-D08 & - & \\
\hline 24 & BVMO-CDX-003 & - & \\
\hline 25 & BVMO-P3-C07 & - & \\
\hline 26 & BVMO-P1-C06 & - & \\
\hline 27 & 2,5-DKCMO & Pseudomonas putida & 2,5 二酮茨烷单加氧酶 \\
\hline 28 & $\mathrm{BVMO}_{\text {Ocean }}$ & Oceanicola batsensis DSM 15984 & \\
\hline 29 & $\mathrm{BVMO}_{\text {Parvi }}$ & Parvibaculum lavamentivorans DSM 13023 & \\
\hline 30 & 3,6-DKCMO & Pseudomonas putida & 3,6 二酮茨烷单加氧酶 \\
\hline 31 & CDMO & Rhodococcus ruber SC1 & 环十二酮单加氧酶 \\
\hline 32 & $\mathrm{CHMO}_{\mathrm{Tm}}$ & Thermocrispum municipale DSM 44069 & 环己酮单加氧酶 \\
\hline 33 & MtmOIV & Streptomyces argillaceus & 光神霉素单加氧酶 \\
\hline 34 & PenE & Streptomyces exfoliatus & \\
\hline 35 & PntE & Streptomyces arenae & \\
\hline 36 & PtIE & Streptomyces avermitilis & \\
\hline 37 & $\mathrm{BVMO}_{\mathrm{AR}}$ & Acinetobacter radioresistens & \\
\hline 38 & FMO CcsB & Aspergillus clavatus NRRL 1 & \\
\hline 39 & $\mathrm{BVMO}_{\mathrm{Bo}}$ & Bradyrhizobium oligotrophicum & \\
\hline 40 & $\mathrm{BVMO}_{\mathrm{Am}}$ & Aeromicrobium marinum & \\
\hline 41 & SMO & Pseudomonas putida $C A-3$ & 苯乙烯单加氧酶 \\
\hline 42 & YMOA & Yarrowia lipolytica & \\
\hline 43 & $\mathrm{CHMO}_{\mathrm{Tm}}$ & Thermocrispum municipale DSM 44069 & \\
\hline 44 & $\mathrm{BVMO}_{\mathrm{Af} 1}$ & A. fumigatus Af293 & \\
\hline
\end{tabular}


化反应研究的新进展.

\section{1 直链酮底物}

BVMO 催化直链酮底物的 BV 氧化, 对以生物催化 的方式生产石油工业相关产品有重大意义. 直链酮底物 的烷基链长度会影响 BVMO 对底物的接受程度, 常见 的环已酮单加氧酶(CHMO)在氧化至多 3 个碳原子的烷 基链时, 表现出高效率和高选择性 ${ }^{[2]}$. Rial 等 ${ }^{[4]}$ 在 2017 年

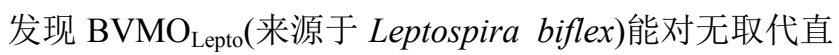
链酮和有支链的直链酮进行生物氧化, 优先生成正常的 内酯产物. 它可以高效转化小于 6 个碳的直链酮, 对于 碳链较长的底物(例如 2-甲基-4 辛酮、2-壬酮、4-壬酮), 只有在较低底物浓度 $(0.1 \mathrm{mg} / \mathrm{mL})$ 时才有较高的转化率. 而来自真核生物的 $\mathrm{BVMO}_{\mathrm{Cm}}$ (来源于 Cyanidioschyzon merolae)和 $\mathrm{BVMO}_{\mathrm{Pp}}$ (来源于 Physcomitrella patens) 对 2壬酮和 3-壬酮等长链酮能实现完全转化 ${ }^{[5]}$. 己酮可可碱 1 是一种广泛使用的药物, hFMO5(来源于 Human)对这 种具有㛶基的药物有很强的体外氧合活性, 可以将其氧 化成相应的乙酸酯, 反应具有高度的区域选择性(30： $1)^{[6]}$ (Scheme 1).

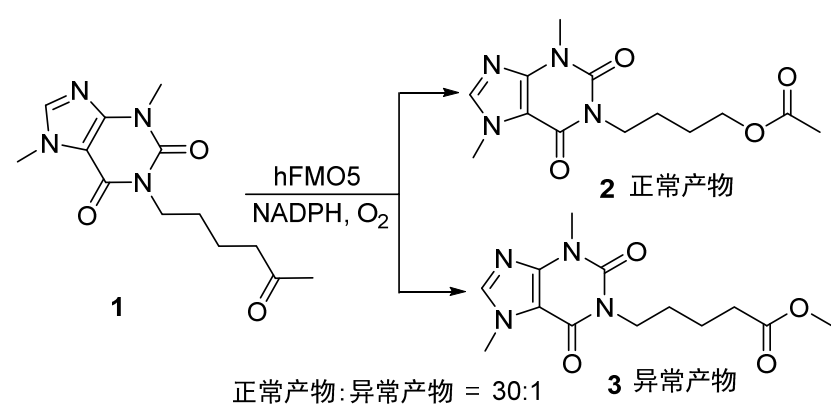

图式 1 hFMO5 催化已酮可可碱的区域选择性 BV 氧化反应

Scheme 1 Regioselective BV oxidation reaction of pentoxifylline catalyzed by hFMO5

为了提高催化效率, 工程化改造的 BVMO 也被用 于氧化直链酮底物. 例如, Mihovilovic 等 ${ }^{[7]}$ 在 2015 年开 发了一种利用 BVMO 将乙酰丙酸酯 4 氧化为 3-羟基丙 酸 6 衍生物的方法. 其中 $\mathrm{CPMO}_{\mathrm{Coma}}$ (来源于 Comamonas testosterone, 环戊酮单加氧酶) 的 F $156 \mathrm{~L}$ 突变株对乙酰丙 酸酯(甲、乙、丁酯)类底物的转化率有明显的提升, 是 生物转化扩大生产过程中的最佳选择(Scheme 2). 为了 改善 $\mathrm{CHMO}_{\text {Acineto }}$ (来源于 Acinetobacter sp. 的环己酮单加 氧(酶)转化 2-丁酮的催化活性以及对丙酸甲酯的区域选 择性, Fraaije 等 ${ }^{[8]}$ 对酶上底物结合位点附近的多个残基 和 $\mathrm{NADP}^{+}$结合位点进行饱和突变, $\mathrm{CHMO}_{\text {Acineto }}$ 的最佳 突变株 T56S/I491A 对 2-丁酮的转化率为 $73 \%$, 产物中 丙酸甲酯的得率为 $43 \%$, 均优于野生型 $(26 \%)$. 后续的
研究表明，以 2-丁醇为原料用醇脱氢酶产生丁酮并以 BVMO 转化为甲酸丁酯和乙酸乙酯的方法也是可行 的 ${ }^{[9]}$. Carvalho 等 ${ }^{[10]}$ 在使用 $\mathrm{PAMO}$ (来源于 Thermobifida fusca 的苯丙酮单加氧酶)四突变株(P253F/G254A/ $\mathrm{R} 258 \mathrm{M} / \mathrm{L} 443 \mathrm{~F}$ ) 对 2-辛酮进行转化时, 其活性远超野生 型, 证明了活性位点的重塑可以改善 PAMO 对长链脂肪 族底物的结合.

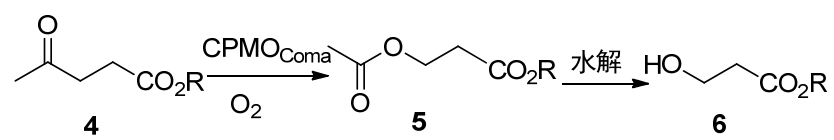

图式 $2 \mathrm{CPMO}_{\mathrm{Coma}}$ 催化乙酰丙酸烷基酯的 $\mathrm{BV}$ 氧化反应

Scheme 2 Bayer-Villiger oxidation reaction of alkyl levulinate catalyzed by $\mathrm{CPMO}_{\text {Coma }}$

此外, BVMO 催化直链酮的氧化反应也被组合进细 胞内的多步级联反应中, 用于生产重要的工业酸. 2013 年, Park 等 ${ }^{[11]}$ 利用多步酶法以油酸 7 为原料催化合成 $\omega$ 差基羧酸 13. 以水合酶和醇脱氢酶将不饱和脂肪酸前 体中的双键氧化为酮，再使用不同区域选择性的 BVMO 催化氧化成酯, 水解后得到 $\alpha, \omega$-二羧酸 12 或 $\omega$ 羟基脂肪酸 13 (Scheme 3). 2016 年他们 ${ }^{[12]}$ 通过使用新的 脂肪酸双键水合酶和大肠杆菌细胞, 提高了脂肪酸向细<smiles>CC(CC=CCC(C)(C)C)C(C)(C)C(=O)O</smiles>

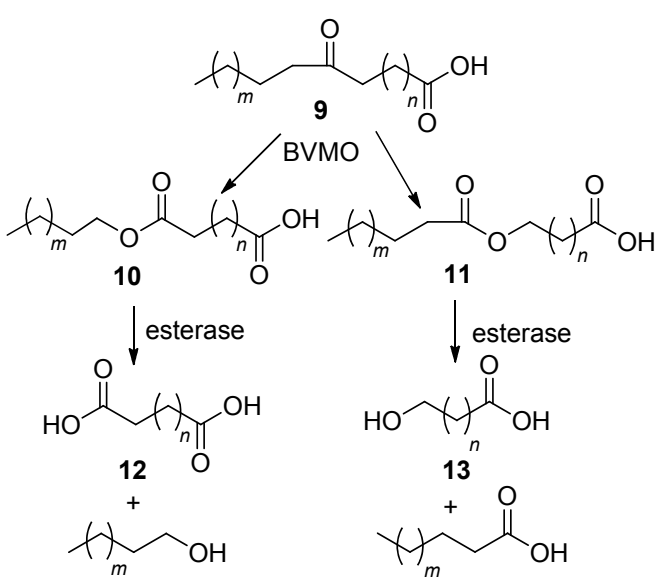

图式 3 通过多步酶催化反应将不饱和脂肪酸转化为 $\alpha, \omega$-二 羧酸或 $\omega$-着基脂肪酸

Scheme 3 Conversion of unsaturated fatty acids to $\alpha, \omega$-dicarboxylic acids or $\omega$-hydroxy fatty acids by multi-step enzymatic reactions 
胞内级联酶转运的速率, 从而实现了一锅法将植物油进 行氧化裂解, 最终转化为 C9 羧酸的级联反应, C9 羧酸 是一种潜在的抗真菌剂. 2018 年 Seo 等 ${ }^{[13]}$ 利用 1,9-壬二 酸仲醇脱氢酶、BVMO、长链伯醇脱氢酶和醛脱氢酶成 功将脂肪酸通过酶/全细胞环境转化为工业相关的 C9 羧 酸(9-着弪基壬酸、正壬酸等).

\section{2 含芳香基团的酮底物}

HAPMO(来源于 Pseudomonas fluorescens ACB 的 4羟基苯乙酮单加氧(酶)是最早发现对含有芳香基团的酮 类有催化活性的 $\mathrm{BVMO}^{[14]}$, 其催化 3-苯基 2-丁酮(14)动 力学拆分时产物 15 的 ee 值可达 99\% (Scheme 4). 2011 年, Fraaije 等 ${ }^{[15]}$ 从微生物 Rhodococcus jostii RHA1 的基 因组中找到 22 种 Type I 型 BVMO, 其中 BVMO9(来源 于 Rhodococcus jostii RHA1) 和 BVMO15(来源于 Rhodococcus jostii RHA1)与 HAPMO 的底物范围相类 似，对多种芳香酮都表现出高活性.

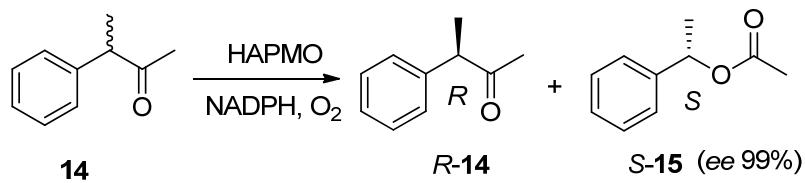

图式 4 HAPMO 催化 3-苯基 2-丁酮的动力学拆分反应 Scheme 4 Kinetic resolution reaction of 3-phenyl 2-butanone catalyzed by HAPMO

含芳香基团的酮类底物中，其酮基团和芳香环的距 离对 BVMO 的催化活性也有影响. 2014 年 Opperman 等 ${ }^{[16]}$ 使用 $\mathrm{BVMO}_{\mathrm{AFL} 456} 、 \mathrm{BVMO}_{\mathrm{AFL} 838} 、 \mathrm{BVMO}_{\mathrm{AFL} 210}$ 和 $\mathrm{BVMO}_{\mathrm{AFL} 619}$ (来源于 Aspergillus flavu NRRL3357)转化 4苯基 -2-丁酮 16 等底物, 其中 $\mathrm{BVMO}_{\mathrm{AFL} 456}$ 和 $\mathrm{BVMO}_{\mathrm{AFL} 619}$ 的活性随着羰基和苯环距离的增加而下降, 而另外两种 BVMO 的活性变化则相反. 此外他们还发 现, 如果苯环的对位上有羟基或甲氧基取代, 也会导致 反应转化率下降(Scheme 5).

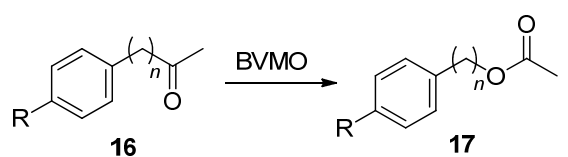

图式 5 BVMO 催化芳香酮的 Baeyer-Villiger 氧化 Scheme 5 Bayer-Villiger oxidation reaction of aromatic ketone catalyzed by BVMO

与 HAPMO 相比, PAMO 同样可以转化芳香酮底物, 在反应活性、立体选择性及热稳定性上都更有优势 ${ }^{[17]}$, 但 PAMO 的底物范围狭窄, 一般只能接受体积小的底 物, 对较大的底物其反应活性和立体选择性都比较 差 $^{[18]}$. 蛋白质工程可以克服 PAMO 的这种缺点, 从而扩
大其底物范围. 例如, PAMO 的 M446G 突变能接受数种 野生型 PAMO 无法转化的大体积底物, 如 1-茚酮(18). 该突变株在催化 1-狮酮的 BV 氧化反应时, 有很高的区 域选择性，只生成异常内酯 1-异苯并二氢吡喃酮(19) (Scheme 6) ${ }^{[19]}$. 此外在 PAMO 的 P253 和 L443 这两个氨 基酸残基上引入苯丙氨酸，也能增加 PAMO 对一些含有 芳环的底物的活性 ${ }^{[20]}$. 由于 STMO(来源于 Rhodococcus rhodochrous 的类固醇单加氧酶) 的活性位点结构和 PAMO 非常相似，因此, Fraaije 和 Mattevi 等 ${ }^{[21]}$ 推测并验 证, STMO 对 PAMO 的天然底物苯丙酮也能起到催化氧 化的作用，针对 STMO 上疏水和亲水残基，并结合其他 几种 BVMO 相关结构特征进行定向进化, 篮选出 STMO 的 V72I 突变对苯丙酮的反应活性是野生型的 3 倍.

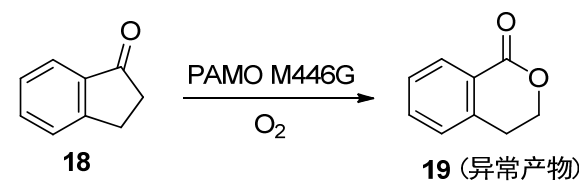

图式 6 通过 PAMO M446G 催化 1-狮酮的 BV 氧化反应 Scheme 6 Bayer-Villiger oxidation reaction of 1-indone catalyzed by PAMO M446G

除了芳香酮的 BV 氧化, BVMO 还能催化含有苯基 的醛氧化反应. BVMO4(来源于 Dietzia sp. D5)对含有苯 基的醛类具有较高的活性，可以将 2-苯基丙醛(20)转化 为 2-苯基丙酸 $(21)$ 和相应的酯产物, 酯产物最终水解生 成 1 -苯基乙醇 $(22)$, 底物低浓度时生成等量的酯和酸, 高浓度时反应趋向于生成酯，而使用 4 倍量的酶时，产 物趋向于生成酸(Scheme 7). 除此之外, BVMO4 还能氧 化长链的脂肪醛, 例如辛醛和癸醛 ${ }^{[22]}$.

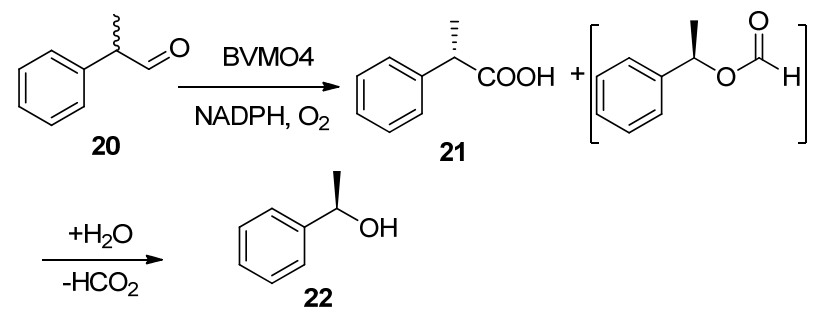

图式 7 BVMO 催化 2-苯基丙醛的 BV 氧化 Scheme 7 Bayer-Villiger oxidation reaction of 2-phenylpropionaldehyde catalyzed by BVMOs

\section{3 单环及双环类酮底物}

\section{1 单萜类底物}

单萜是高性能再生聚合物的单体原料 ${ }^{[23]}$, 在 BVMO 生物氧化研究中, 往往用于酶的区域选择性研 究(Scheme 8). 许多野生型的 BVMO 本身就拥有很高的 
区域选择性, 往往只生成一种区域异构体。例如, $\mathrm{CHMO}_{\mathrm{JS} 666}$ (来源于 Polaromonas sp. strain JS666 的环己 酮单加氧酶)能缓慢将 (十)-香芹酮 23 完全转化为相应的 异常内酯 $(0: 100)^{[24]}$. CPDMO(来源于 Pseudomonas sp. $\mathrm{HI}-70$ 的环十五酮单加氧酶)在转化这类底物时, 几乎只 产生一种区域异构体, 并有着优异的立体选择性(ee> 99\%). 其中(一)-薄荷酮 24 是首次被 BVMO 转化, 只生 成正常的产物 ${ }^{[25]}$. CAMO(来源于 Cylindrocarpon radicicola ATCC 11011 的环烷酮单加氧酶) 和 OTEMO(来源于 Pseudomonas putida NCIMB 10007)在 转化这类底物时, 后者有更强的区域选择性, OTEMO 在转化(一)-二氢香芹酮 (25)和 $(+)$-薄荷酩(26)时只产生 正常内酯产物 ${ }^{[26]}$.

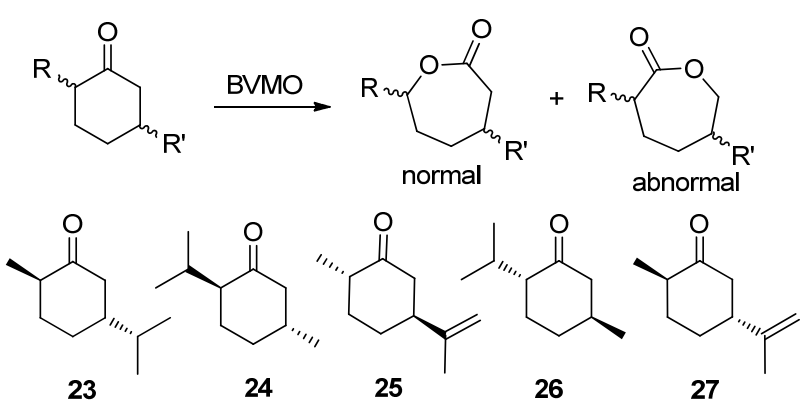

图式 8 BVMO 催化萜烯酮的区域选择性氧化

Scheme 8 Regioselective oxidation reaction of terpeneone catalyzed by BVMO

在许多情况下, BVMO 缺乏完美的区域选择性. BVMO 催化氧化的区域选择性主要取决于活性位点的 手性环境和迁移取代基的电子效应, 对活性位点的残基 进行突变, 可以改进 BVMO 的区域选择性. Bornscheuer 等 ${ }^{[27]}$ 利用理性设计得到 $\mathrm{CHMO}_{\text {Arthro }}$ 的 F299A/F330A/ F485A 突变株来转化 $(+)$ 二氢香芹酮 27, 使底物倒置结 合, 产物中正常和异常内酯的比例为 $99: 1$, 而野生型 酶只生成异常产物. 为了证明这种设计的适用性, 他 们 ${ }^{[27]}$ 又将突变引入有相似活性位点的 $\mathrm{CHMO}_{\mathrm{Acineto}}$, 从 中篮选出 $\mathrm{CHMO}_{\text {Acineto }}$ F246V/F277A/F432A 突变株, 这 种突变株只产生异常内酯 $(0: 100)$, 再次完全反转了相 应野生型酶的区域选择性(Scheme 9). 类似的区域选择 性反转的结果在 $\mathrm{CHMO}_{\text {Phil }}$ (来源于 Rhodococcus sp. Phi1)酶中也得以实现, 野生型 $\mathrm{CHMO}_{\text {Phil }}$ 催化时只形成 异常产物, 而 CHMO $_{\text {Phi1 }}$ F299A/F330A/F485A 突变株则 只生成正常产物 ${ }^{[23]}$.

\section{2 环丁酮及其衍生物}

环丁酮类底物是少数几种仅使用过渡金属催化 $\mathrm{BV}$ 氧化即可获得高立体选择性产物的底物 ${ }^{[28]}$, 相应的酯 产物具有广泛的生物学特性, 可用作香料和香气成分以

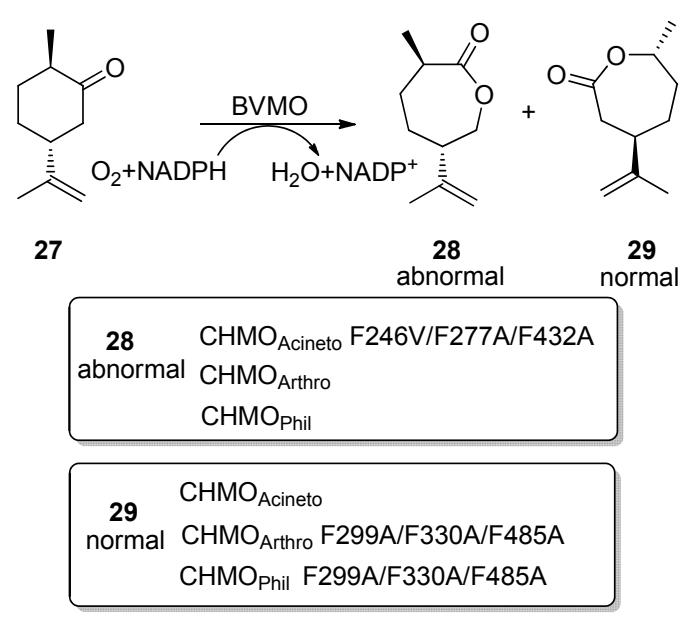

图式 9 几种 $\mathrm{CHMO}$ 及其突变株催化 $(+)$ 二氢香芹酮的 BV 氧 化反应

Scheme 9 Bayer-Villiger oxidation reaction of (+)-dihydrocarvone catalyzed by $\mathrm{CHMO}$ and variants

及生物燃料, 也是合成很多具有复杂结构的天然产物的 重要中间体. 使用 BVMO 转化前手性环丁酮, 过程更环 保，适用性也更高. 来自美国 Codexis 公司的 6 种 $\mathrm{BVMO}^{[29]}$ 和 $\mathrm{CHMO}_{\mathrm{JS} 666}{ }^{[24]}$ 可以催化 3-取代环丁酩的去 对称反应获得 $\gamma$-丁内酯衍生物, 其中 BVMO-CDX-

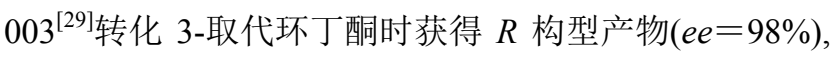
而 BVMO-P3-C07 $7^{[29]}$ 和 BVMO-P1-C06 $6^{[29]}$ 则主要生成 $S$ 构型产物 ${ }^{[29]}$. Mihovilovic 和 Bornscheuer 等 ${ }^{[26]}$ 在 2016 年 以 1,3-丁二烯为起点, 用 CAMO 和 OTEMO 催化 3-取代 前手性环丁酮的去对称氧化反应, 最终生成 $(R)-(-)-$ Taniguchi 内酯(30) (Scheme 10).

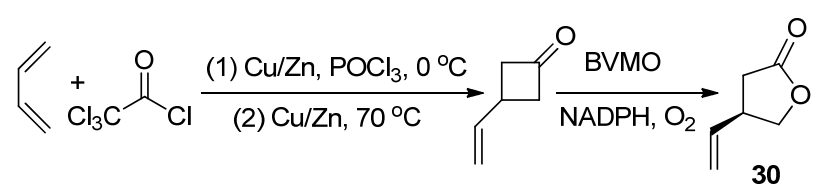

图式 10 以 1,3-丁二烯为起点通过 BVMO 生成 Taniguchi 内 酯

Scheme 10 Synthesis of taniguchi lactone by BVMO with 1,3butadiene as the starting material

\section{3 环己酮及其衍生物}

环己酮及其衍生物是非常重要的化工原料, 也是目 前 BVMO 研究中使用最广泛的底物之一.

\subsubsection{4-取代环已酮}

4-取代环己酮本身并无手性，但在 BVMO 催化的 去对称氧化过程中能生成手性产物. 通过对 BVMO 酶 的特定位点进行定向进化可以增强或逆转 BVMO 的立 体选择性. 2004 年由 Reetz 等 ${ }^{[30]}$ 首次报道了相关研究. 他们利用 CHMO 突变株 F432S 催化 4-羟基环己酮, 反 
应的立体选择性由野生型的 $e e=9 \%(R)$ 翻转至 $e e=79 \%$ $(S)$. 之后他们通过 4-甲基-环已酮 ${ }^{[31}$ 和 4-差基-环己酮的 $\mathrm{QM} / \mathrm{MM}$ 计算研究探讨了 BVMO 的催化机理, 发现反应 的立体选择性可能与去质子化的黄素氢过氧化物以及 阴离子形式的 Criegee 中间体有关 ${ }^{[32]}$. 许多天然与非天 然有机烯烃化合物, 其相应的双键具有 $E$-或 $Z$-构型. Reetz 等发现野生型 $\mathrm{CHMO}_{\text {Acineto }}$ 在全细胞环境下催化 4亚甲基环己酮衍生物 31 的去对称反应时, 产物中 $E$ 构 型和 $Z$ 构型比例为 $96: 4$. 之后他们通过 3 轮的 ISM (Iterative Saturation Mutagenesis, 迭代饱和突变)进化策 略, 篎选出最佳 $\mathrm{CHMO}_{\text {Acineto }}$ 的突变株 F432I/T433G/ L143M/F505C, 它能完全反转这类底物的立体选择性 $(E: Z=4: 96)$, 同时其转化率可达 $98 \%{ }^{[33]}$ (Scheme 11). PAMO 野生型无法接受 4-取代环己酮作为底物, 通过定 向进化篮选出来的 PAMO (I67A/R258A/A442P/ S444A/P286R/L443F/Y502F) 突变株可以催化 4-(溴代亚 甲基)-环已酮, 4-甲基环己酮的去对称反应 ${ }^{[34]}$, PAMO 的 Q93N/P94D 突变株在转化 4-烷基环己酮时也展现出很 高的对映选择性 ${ }^{[35]}$. 此外例如 $\mathrm{CAMO}^{[26]} 、 \mathrm{CHMO}_{\text {JS666 }}{ }^{[24]}$ 、 $\mathrm{CPDMO}^{[25]}$ 以及 $\mathrm{OTEMO}^{[26]}$ 这些野生型 BVMO 也都能 催化 4-取代环己酮的去对称反应, 并具有优异的立体选 择性, 其中 OTEMO 在转化 4-甲酸乙酯-环己酮时, 可以 获得 $e e$ 值 $>96 \%$ 的立体选择性, 超越所有已知野生型 $\mathrm{BVMO}^{[26]}$.<smiles>CCOC=C1CCC(=O)CC1</smiles>

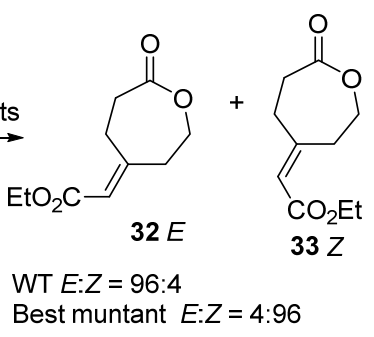

图式 11 由 $\mathrm{CHMO}_{\text {Acineto }}$ 及其突变株催化 4-亚甲基环己酮衍生 物的去对称反应

Scheme 11 Diastereoselective desymmetrization of 4-methylene cyclohexanones by WT $\mathrm{CHMO}_{\text {Acineto }}$ and mutants

\subsubsection{2-取代环已酮}

通过 BVMO 催化 2-取代环己酮的动力学拆分反应, 能提供高纯度的手性内酯产物. 与上述 4-取代环己酮类 似，通过对活性位点周围的特定残基进行定向进化可以 控制拆分反应的立体选择性. Reetz 等 $^{[35]}$ 在 2010 年根据 变构效应对 PAMO 远离底物结合口袋的位点进行饱和 突变, 经篮选 400 个突变株得到 PAMO Q93N/P94D 突 变株, 这种突变株在催化 2-取代环已酮的不对称动力学 氧化拆分反应时有优异的立体选择性. Berghuis 等 $^{[36]}$ 在 2014 年发现 CHMO W492A 突变株在转化 2-取代环己酮
时比起野生型有更高的 $(S)$-构型立体选择性. 与 Fre(来 源于 Escherichia coli 的黄素还原酶)共同表达的 2,5-DKCMO (2,5 二酮茨烷单加氧酶)在转化 2-苯基环己 酮时，其选择性因子 $E>200(R)^{[37]}$. CHMO 的热稳定型 突变株 DS4 (Y411C, A463C)对 2-甲基环己酮进行拆分 时能得到 $98 \%(R)$ 的底物 $e e$ 值, 远超野生型的立体选择 性 ${ }^{[38]}$. 此外 $\mathrm{CPDMO}^{[25]} 、 \mathrm{CAMO}^{[26]}$ 以及 $\mathrm{OTEMO}^{[26]}$ 这些 野生型 BVMO 也能催化 2-取代环己酮的动力学拆分反 应，后 2 种酶的转化产物优势构型相同，但 CAMO 的立 体选择性更佳.

\subsection{3 其他环已酮衍生物}

$\varepsilon$-己内酯 36 是一种非常重要的工业原料, 广泛用于 聚合物生产, 每年产量可达数万吨. 化学法通常用过氧 乙酸作为氧化剂进行生产, 但存在有毒性并在生态学和 安全性上有诸多缺点. 近几年 Gröger, Bornscheuer 和 Liese 等课题组聚焦于此, 发明了一系列以环己醇为起 点生产 $\varepsilon$-己内酯 36 的级联反应(Scheme 12). 通过使用 $\operatorname{PDH}(\text { 多元醇脱氢 酶 })^{[39]} 、 \mathrm{ADH}(\text { 醇脱氢 酶 })^{[40]}$ 和 LK-ADH(来源于 Lactobacillus kefir 的醇脱氢酶)等酶催 化剂 ${ }^{[41]}$, 利用空气中的氧气可以将环己醇 34 氧化为环 己酮 35, 同时将 $\mathrm{NADP}^{+}$还原为 $\mathrm{NADPH}$ ，再使用 $\mathrm{CHMO}$ 将环己酮氧化为 $\varepsilon$-己内酯衍生物. 该过程不需要额外的 共底物进行辅因子再生. 2015 年, 他们 ${ }^{[42]}$ 又利用 CAL-A(南极假丝酵母的脂肪酶 A) 使 $\varepsilon$-己内酯衍生物开 环聚合，有效地解决了产物抑制的问题. 2017 年, Rudroff 等 ${ }^{[43]}$ 建立了 Michaelis-Menten 参数的模型, 证明 了这个级联反应的瓶颈在于反应中 BVMO 的活性. 而 Kohl 等 ${ }^{[44]}$ 在这类级联反应中对 $\mathrm{CHMO}$ 和 $\mathrm{ADH}$ 使用共 表达策略，比起单独表达能实现更高效的辅因子循环， 从而获得更高的转化率.

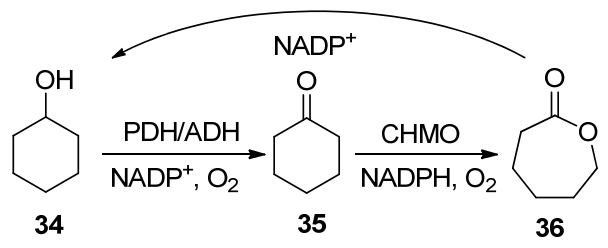

图式 12 以环己醇为原料利用醇脱氢酶和 $\mathrm{CHMO}$ 生产 $\varepsilon$-己内 酯的多步酶法级联反应

Scheme 12 Multi-step cascade reaction using cyclohexanol as starting material to produce $\varepsilon$-caprolactone by $\mathrm{ADH}$ and $\mathrm{CHMO}$

一般来说, 烯酮不会被 BVMO 转化, Alphand 等 ${ }^{[45]}$ 在 2014 年发现 2 种新型 BVMO 可以转化烯酮为不饱和 内酯. $\mathrm{BVMO}_{\text {Ocean }}$ (来源于 Oceanicola batsensis DSM 15984)可以在环己(庚、辛)烯酮 38 的羰基与非烯属碳原 子之间插入氧原子, 生成共轭烯内酯 37 , 而 $\mathrm{BVMO}_{\text {Parv }}$ (来源于 Parvibaculum lavamentivorans DSM 13023)则 
可以在羰基和双键之间插入氧原子生成烯醇内酯 39 (Scheme 13). 另外这两种酶在全细胞环境中转化 3-甲基 环己酮和 4-甲基环己酮获得高光学纯度的内酯产物(ee $98 \%$ ).

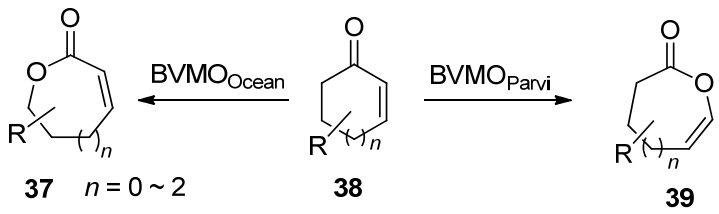

图式 $13 \mathrm{BVMO}_{\text {Ocean }}$ 和 $\mathrm{BVMO}$ Parv 对 $\alpha, \beta$ 不饱和环酮的生物转 化

Scheme 13 Biotransformation of $\alpha, \beta$-unsaturated cyclic ketones catalyzed by $\mathrm{BVMO}_{\text {Ocean }}$ and $\mathrm{BVMO}_{\text {Parvi }}$

$\alpha, \alpha$-二烷基环酮的 BV 转化具备生成季碳手性中心 内酯的潜力. Procter 等 ${ }^{[46]}$ 发明了一种以 $\mathrm{CHMO}_{\text {Acineto }}$ 作 为催化剂, 对带有 $\alpha$-季碳手性中心的外消旋五元或六元 环酮 40 进行动力学拆分的反应. 这种反应同时具备高 立体选择性和化学选择性, 可以用于生产含有季碳手性 中心的不饱和内酯, 并通过 $\mathrm{SmI}_{2}$ 介导的环化作用生成 高立体选择性的 1,4-环庚(辛)二醇 42 (ee > 99\%) (Scheme 14).

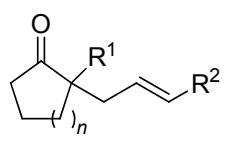

$n=1,2$

40

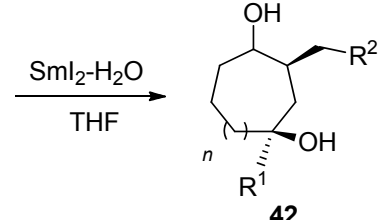

图式 14 具有 $\alpha$-季碳手性中心的外消旋环酮在 $\mathrm{CHMO}_{\text {Acineto }}$ 和 $\mathrm{SmI}_{2}$ 的催化下生成 1,4-环庚(辛)二醇

Scheme 14 A biocatalytic-chemical approach to complex, medium-sized cycloheptane/cyclooctanone-1,4-diol

\section{4 双环酮底物}

\subsection{1 茨酮及其衍生物}

茨酮是一种双环单萜类的化合物, 在自然界存在 2 种对映异构体，(十)莰酮 43 来源于樟树，(一)茨䣶 44 可 在植物精油中发现. 目前已知有三种 BVMOs 参与荻酮 降解途径, 分别是 2,5-DKCMO (2,5-二酮茨烷单加氧 酶)、3,6-DKCMO 酶(3,6-二酮茨烷单加氧酶) 以及 OTEMO. 前 2 者属于 Type II 型的 BVMO, 这类 BVMO 的辅因子是比起 NADPH 便宜很多的 NADH, 因此有较 高的工业应用价值 ${ }^{[1]}$. 其中 $2,5-\mathrm{DKCMO}$ 能转化 (一)莰酮 44, 但转化率不如 $(+)$ 葫酮 43, 而 3,6-DKCMO
在转化这 2 种底物时差异不大, 对 (一)茨酮 44 转化率要 稍好一些 ${ }^{[47]}$. 而 OTEMO 对 $(+)$ 莰酮 43 的转化率高于 (一)莰酮 $\mathbf{4 4}^{[37]}$. 除此以外, $\mathrm{CDMO}$ (来源于 Rhodococcus ruber SC1 的环十二酮单加氧酶 $)^{[48]}$ 和 $\mathrm{CAMO}^{[49]}$ 也能转 化这类底物, 前者还能转化降樟脑 $\mathbf{4 5}$, 且有优异立体选 择性.

对 BVMO 和相关辅酶基因采取共表达策略，是一 种增强反应活性的手段. 例如, Lau 等 ${ }^{[37]}$ 在 2013 年将来 自 2,5-DKCMO 和 3,6-DKCMO 的 3 个关于茨酮降解的 功能性基因和 Fre 的基因串联, 在无细胞环境下转化 $(+)$ 茨酮 43/(一)茨酩 44, 发现 2,5-DKCMO 的反应活性 增加了近 20 倍. 而将 Fre 基因与 2,5-/3,6-DKCMO 共表 达, 能显著增强这 2 种酶对茨酮和降樟脑的转化能力, 但如将 2 种基因融合到同一个阅读框架进行表达，则会 降低转化率 ${ }^{[50]}$.<smiles>CC12CCC(C)(C1)C(=O)C2</smiles>

43

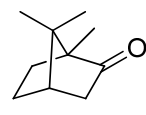

44

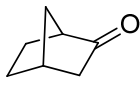

45

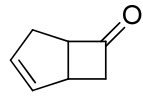

46

\subsection{2 双环 $[3,2,0]$-庚-2-烯-6-酮}

消旋双环 [3.2.0]-庚-2-烯-6-酮(46) 是一种常用于研 究 BVMO 的立体和区域选择性的经典底物(Scheme 15). 该底物不容易发生副反应，以插入氧原子位置不同而产 生普通/异常产物的比例来衡量酶的区域选择能力(表 2).

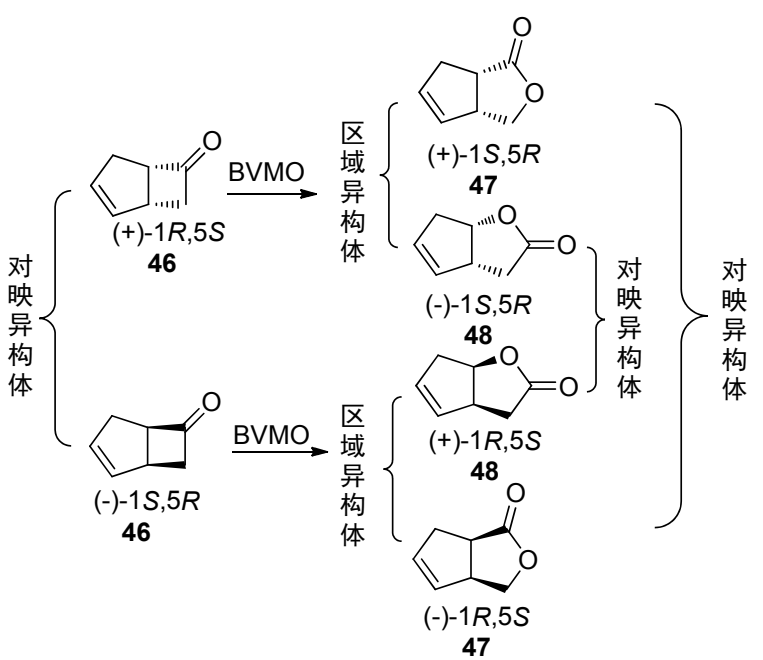

图式 15 BVMO 催化双环 $[3,2,0]$ 庚-2-烯-6-酮发生 BV 氧化反 应

Scheme 15 Bayer-Villiger oxidation reaction of bicyclo[3,2,0]hept-2-en-6-one catalyzed by BVMOs

野生型 PAMO 在转化这种底物时, 产物中正常内酯 48 和异常内酯 47 的比例约为 $3: 1$. 而 PAMO 的 $\mathrm{A} 435 \mathrm{Y} 、 \mathrm{~A} 442 \mathrm{G}$ 和 $\mathrm{Q} 152 \mathrm{~F}$ 这 3 种突变株 ${ }^{[19]}$ 则展示了与 
表 2 各种 BVMO 催化[3.2.0]-庚-2-烯-6-酮的 BV 氧化反应区域选择性

Table 2 Regioselectivity of Bayer-Villiger oxidation of [3.2.0]-hept-2-en-6-one catalyzed by BVMOs

\begin{tabular}{|c|c|c|c|c|c|}
\hline Entry & BVMO & 46 & Conv. $/ \%$ & $n: a^{a}$ & Ref. \\
\hline 1 & $\mathrm{CHMO}_{\text {Acineto }}$ & rac-46 & $>99$ & $60: 40$ & [38] \\
\hline 2 & CHMO DS2 (N286C, A293C) & $r a c-46$ & 87 & $60: 40$ & {$[38]$} \\
\hline 3 & CHMO DS3 (T415C, A463C) & $r a c-46$ & $>99$ & $60: 40$ & [38] \\
\hline 4 & CHMO DS4 (Y411C, A463C) & rac-46 & 28 & $65: 35$ & [38] \\
\hline 5 & CHMO T415C & rac-46 & $>99$ & $62: 38$ & [38] \\
\hline 6 & $\mathrm{CHMO}_{\mathrm{Tm}}$ & rac-46 & 100 & $49: 51$ & [54] \\
\hline 7 & BVMOAFL210 & rac-46 & 56 & $87: 13$ & [16] \\
\hline 8 & BVMOAFL456 & rac-46 & 53 & $94: 0$ & [16] \\
\hline 9 & BVMOAFL 838 & rac-46 & - & $62: 13$ & [16] \\
\hline 10 & PAMO & rac-46 & - & $73: 27$ & [19] \\
\hline 11 & PAMO Q152F & rac-46 & - & $30: 70$ & [19] \\
\hline 12 & PAMO A435Y & rac-46 & - & $29: 71$ & [19] \\
\hline 13 & PAMO A442G & $r a c-46$ & - & $30: 70$ & [19] \\
\hline 14 & PAMO Q152F/S441A/A442G & $r a c-46$ & - & $23: 77$ & [19] \\
\hline 15 & STMO & rac-46 & - & $65: 35$ & {$[55]$} \\
\hline 16 & PASTMO & rac-46 & - & $66: 34$ & {$[55]$} \\
\hline 17 & PACHMO & rac-46 & - & $39: 61$ & {$[55]$} \\
\hline 18 & PAMEMO1 & rac-46 & - & $76: 24$ & [55] \\
\hline 19 & 2,5-DKCMO-1-Fred & rac-46 & 100 & $57: 43$ & {$[37]$} \\
\hline 20 & 2,5-DKCMO-2-Fred & rac-46 & 100 & $52: 48$ & [37] \\
\hline 21 & 3,6-DKCMO-Fred & rac-46 & 53 & $40: 60$ & [37] \\
\hline 22 & 2.5-DKCMO & rac-46 & - & $57: 43$ & {$[37]$} \\
\hline 23 & OTEMO & rac-46 & $>90$ & $70: 30$ & [26] \\
\hline 24 & CAMO & rac-46 & $>90$ & $53: 47$ & [26] \\
\hline 25 & CPMO & rac-46 & $>90$ & $97: 3$ & [26] \\
\hline \multirow[t]{2}{*}{26} & $\mathrm{BVMO}_{\mathrm{Cm}}$ & $(+)-46$ & 100 & $73: 27$ & {$[5]$} \\
\hline & $\mathrm{BVMO}_{\mathrm{Cm}}$ & $(-)-46$ & 100 & $88: 12$ & {$[5]$} \\
\hline \multirow[t]{2}{*}{27} & $\mathrm{BVMO}_{\mathrm{Pp}}$ & $(+)-46$ & 100 & $76: 27$ & {$[5]$} \\
\hline & $\mathrm{BVMO}_{\mathrm{Pp}}$ & $(-)-46$ & 66 & $62: 4$ & {$[5]$} \\
\hline \multirow[t]{2}{*}{28} & $\mathrm{BVMO}_{\mathrm{Pp}} \mathrm{Y} 160 \mathrm{H}$ & $(+)-46$ & 100 & $72: 28$ & {$[5]$} \\
\hline & $\mathrm{BVMO}_{\mathrm{Pp}} \mathrm{Y} 160 \mathrm{H}$ & $(-)-46$ & 100 & $95: 5$ & {$[5]$} \\
\hline 29 & OTEMO & $(-)-46$ & - & $51: 49$ & {$[52]$} \\
\hline 30 & OTEMO F255V/F443V & $r a c-46$ & 95 & $91: 9$ & {$[52]$} \\
\hline 31 & OTEMO W501A & $(-)-46$ & - & $3: 97$ & {$[52]$} \\
\hline 32 & $\mathrm{BVMO}_{\text {Lepto }}$ & $r a c-46$ & 94 & $50: 50$ & {$[4]$} \\
\hline
\end{tabular}

${ }^{a}$ n: Normal product, a: Abnormal product.

野生型相反的区域选择性 $(\mathbf{4 8}: \mathbf{4 7}=1: 3)$, 其中 PAMO A435Y 转化所得的异常产物 47 还有着优异的立体选择 性(ee 90\%). 而 $\mathrm{CHMO}^{[51]} 、$ PAMO 的 V54I 和 I67T 突变 株 ${ }^{[19]} 、 \mathrm{CAMO}^{[26]}$ 以及 $\mathrm{BVMO}_{\text {Lepto }}$ (来源于 Leptospira

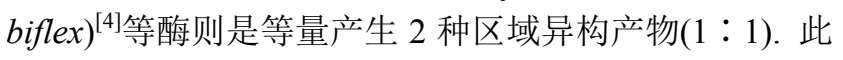
外, OTEMO 的 F255A/F443V 突变株在转化这种底物时 能产生 $90 \%$ 的正常产物 48, 而 W501V 突变株能产生 98\%的异常产物 47, 这 2 种突变株大幅提升了 OTEMO 的区域选择性(野生型为 $1: 1)^{[52]}$. 现如今测序和生物信 息技术逐渐发展成熟，大量新型的 BVMO 已经被发现， 这种底物也用于判断微生物是否有 BVMO 活性. Alphand 等 ${ }^{[33]}$ 在 2015 年使用来自 46 个属的 107 个真菌来 转化该底物, 发现其中 86 种有活性, 证明在真菌中 BVMO 基因序列是普遍存在的.

\section{4 多环以及大分子底物}

多环酮及大分子底物往往是生产抗生素以及激素 的原料或前体. 研究这类化合物的生物转化, 可以为药 物研发和生产提供技术支持. Kurina-Sanz 等 ${ }^{[56]}$ 在 2016 年发现一种曲霉属的微生物(A. parasiticus) 可以转化脱 氢表雄甾酮(DHEA) 49, 通过 D 环的 BV 氧化反应形成 内酯产物, 也可以通过生物氧化途径降解可的松 51 的 侧链, 完全转化为肾上腺甾酮 52, 这说明该微生物中应 该有 BVMO 活性(Scheme 16).

Rohr 等 ${ }^{\left[{ }^{[7]}\right.}$ 在 2013 通过分析对比 MtmOIV(来源于 Streptomyces argillaceus ATCC 12956 的光神霉素单加氧 酶)的晶体结构后, 将底物识别和催化相关的位点逐一 突变为丙氨酸, 在转化前光神霰素 B (Premithramycin 
<smiles>CC12CCC3C(CC=C4CC(O)CCC43C)C1CCC(=O)C2</smiles>

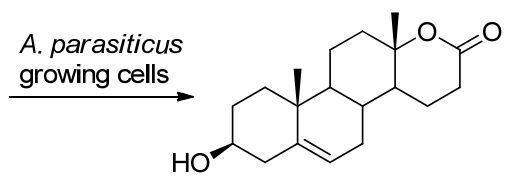

50

51

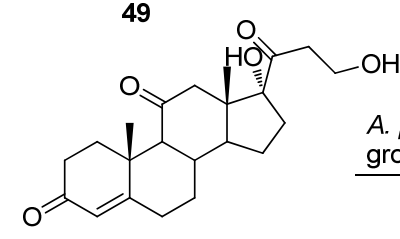

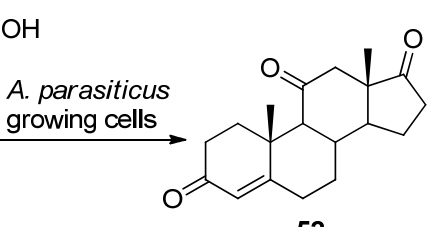

52
图式 16 微生物 A. parasiticus 催化 DHEA 与可的松的转化 Scheme 16 Transformation of DHEA and Cortisone catalyzed by $A$. parasiticus growing cells.

B) 53 时, 发现 P84A 和 F89A 突变相比野生型分别提高 了 1.5 和 5 倍的催化活性, 而 P84A/F89A 突变的催化活 性只能提高 3 倍, 这说明合并多个位点的优势突变其催 化特性未必优于单个位点的优势突变.

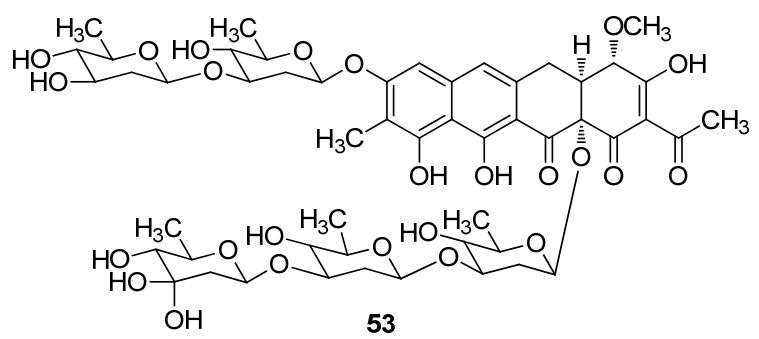

Zhu 等 ${ }^{[58]}$ 在 2015 年利用 1-脱氧-11-氧代戊酸(1deoxy-11-oxopentalenic acid) 54 来考察 PenE(来源于 Streptomyces exfoliatus)、PntE( 来源于 Streptomyces arenae) 以及 $\mathrm{PtlE}$ (来源于 Streptomyces arenae)的区域选 择性, 前 2 种 BVMO 主要生成异常内酯产物(戊烯内酯 $\mathrm{D}$, 55), 而 PtlE 则生成正常内酯(新戊烯内酯 D, 56). 此 外他们利用理性设计得到 PntE 的 13 种突变株, 发现其 中最佳的突变株 L185S 能逆转野生型 PntE 的区域选择 性, 生成正常内酯(Scheme 17).

亚胺培南(Imipenem) 57 是一种碳青霉烯类的抗生 素, 通过检测 $\mathrm{NADPH}$ 消耗实验, 确认了 $\mathrm{BVMO}_{\mathrm{AR}}$ (来源 于 Acinetobacter radioresistens) 可以转化这种 $\mathrm{D}$ 环上带 有 $\mathrm{N}$ 和 $\mathrm{S}$ 元素的底物. 亚胺培南会因碳青霉烯环的 $\mathrm{BV}$ 氧化而插入氧原子, 从而失去抗生素特性, 它是除四环 素以外第二种可以用 BVMO 灭活的抗菌素 (Scheme $18)^{[59]}$.

细胞松弛素 E (Cytochalasin E) 63 是一种血管生成 抑制剂, 2011 年 Tang 等 ${ }^{[60]}$ 在微生物 Aspergillus clavatus NRRL 1 的序列中找到可能参与细胞松弛素生物合成的 $c c s$ 基因簇, 其序列和 CPDMO 表现出高度的同一性 $(41 \%)$ 且有 BVMO 的指纹序列, 于是假定 FMO CcsB(来

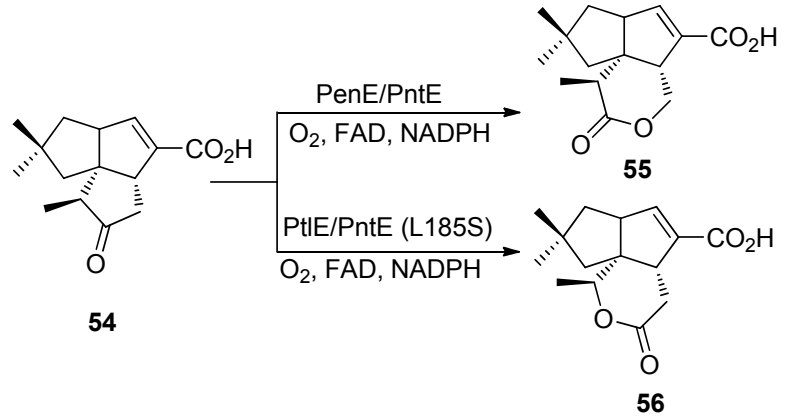

图式 17 PtlE 和 PntE 及其突变株催化 1-脱氧-11-氧代戊酸的 区域选择性氧化

Scheme 17 Regioselective oxidation of 1-deoxy-11-oxopentalenicacid catalyzed by wild type PtlE, PntE and L185S mutant

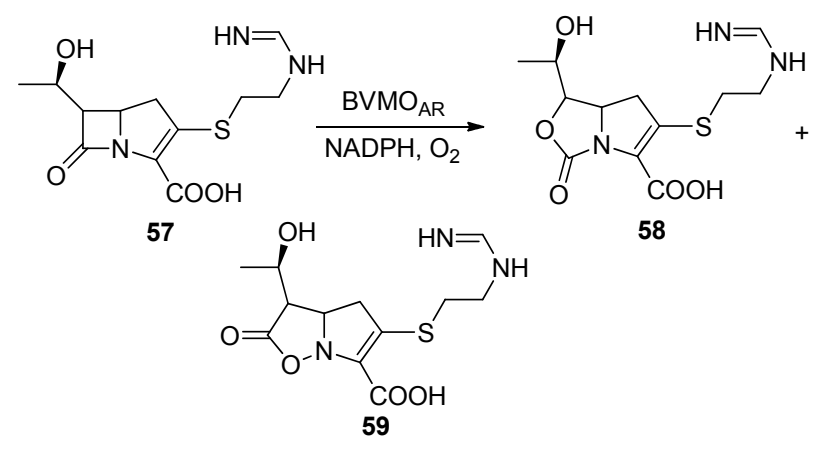

图式 18 BVMO 催化亚胺培南的区域选择氧化反应 Scheme 18 Regioselective oxidation of imipenem catalyzed by BVMO

源于 Aspergillus clavatus NRRL 1 ccs 生物基因簇)的催 化能力与 CPDMO 相似, 这种 BVMO 可能导致两个连续 的 BV 氧化, 从而生成细胞松弛素 E 63 (Scheme 19). 2014 年, 他们 ${ }^{[61]}$ 通过 FMO CcsB 催化细胞松弛素 Z16 的转化, 证实了上述假设. FMO CcsB 的发现提供了将 酮转化为碳酸盐的潜在合成策略.

\section{5 含杂原子底物}

BVMO 除了催化羰基的亲核氧化以外还能实现杂 原子的亲电氧化 ${ }^{[62]}$, 主要以含硼、硒、硫等杂原子的有 机化合物为主. 酶催化杂原子底物有机反应一般分为 2 种, 在杂原子处发生酶促转化, 以及在有机底物的其他 官能团中发生酶促转化, 本综述中主要讨论前者.

\section{1 含嗍有机化合物}

早在 1985 年, Walsh 等[63]就发现了 Type I 型的 BVMO 可以转化含嗍有机化合物. Gonzalo 等 ${ }^{[64]}$ 相继发 现了 PAMO 可以将苯基硼酸氧化成苯酚, HAPMO 可以 氧化多种含嗍苯乙酮衍生物、乙烯基硼化合物以及外消 旋含嗍化合物 ${ }^{[62]}$. PAMO 在转化 3-取代的含嗍苯乙酮衍 生物时, 硼氧化要优先于酮的 BV 氧化和环氧化 ${ }^{[65]}$. 


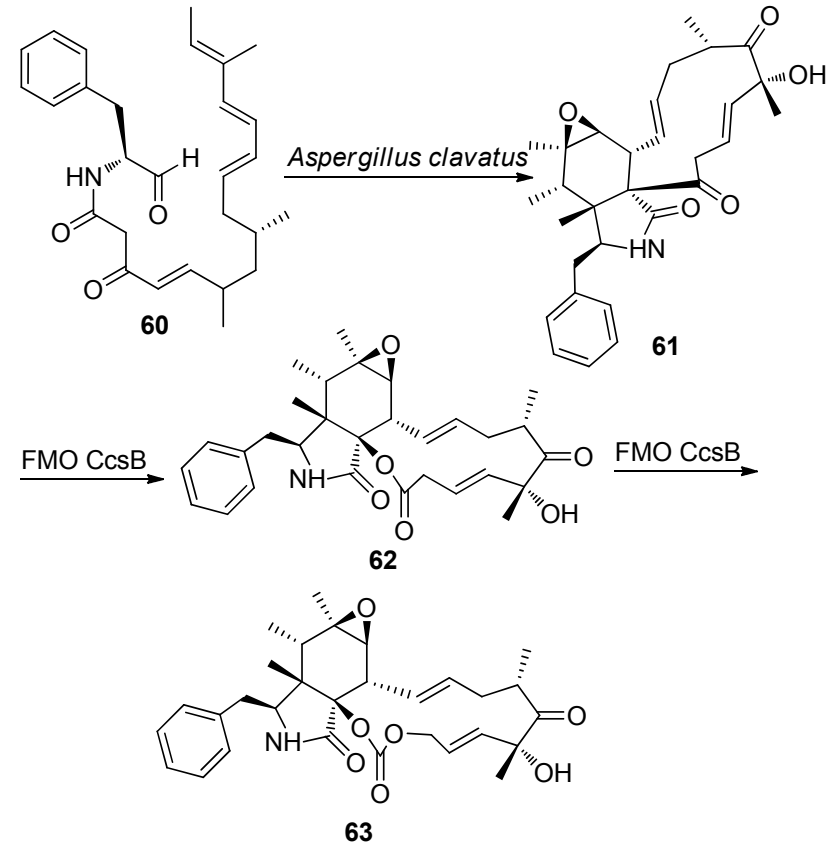

图式 19 BVMOs 参与细胞松弛素 $\mathrm{E}$ 的生物合成途径

Scheme 19 Involvement of BVMOs in the biosynthetic pathways of cytochalasin $\mathrm{E}$

Fraaije 等 ${ }^{[66]}$ 在 2011 年开发了一种以非手性芳族、乙烯 基嗍化合物以及外消旋嗍化合物作为底物，利用 BVMO 对其中的碳硼键进行氧化并通过消除砋酸来得 到相应醇的方法. 在利用 PAMO 及其 $\mathrm{M} 446 \mathrm{G}$ 突变株进 行催化时, 所有底物都产生了嗍氧化反应, 仅 4-取代的 底物出现了酮的 BV 氧化, 而使用 HAPMO 则同时观察 到 2 种氧化反应的发生, CHMO 倾向于碳硼键氧化, 但 反应活性低下. 另外 PAMO 在有机含嗍化合物 64 的动 力学拆分中, 在无细胞环境且 $\mathrm{pH}$ 为 7.5 的条件下, 产物 65 的 $e e$ 值可达 $91 \%(S)$ (Scheme 20). 关于有机嗍化合物 生物转化的报道相对比较少, 主要是因为这些化合物可 能对酶产生抑制作用 ${ }^{[67]}$.

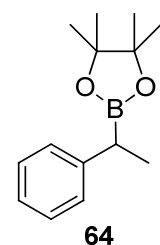

$\mathrm{Ph}=7.5$

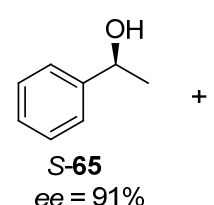

ee $=91 \%$

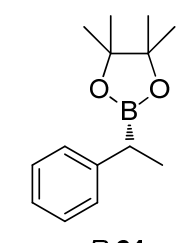

$R-64$
$e e=85 \%$
图式 20 PAMO 催化含嗍化合物的动力学拆分

Scheme 20 Enzymatic kinetic resolution of racemic boroncompounds catalyzed by PAMO

\section{2 含硫有机化合物}

通过化学方法很难实现硫醚的选择性亚砜化, 因此 酶介导的选择性亚砜化在过去几十年来都是有机化学 家关注的热点之一 ${ }^{[65]}$. 该反应的主要问题是如何避免
进一步氧化生成无手性的砜(Scheme 21) ${ }^{[68]}$. 苯烷基硫 醚作为含硫的模型底物, 能够通过 BVMO 转化为相应 的亚砜和砜，广泛地用于 BVMO 研究中。例如, $\mathrm{BVMO}_{\mathrm{AFL} 210}{ }^{[16]} 、 \mathrm{BVMO}_{\mathrm{AFL} 619}{ }^{[16]}$ 和 $\mathrm{BVMO} 4^{[22]}$ 对这类底 物都有较强的活性，但其催化属性各有不同。其中 $\mathrm{BVMO}_{\mathrm{AFL} 210}$ 能进一步将产生的亚砜氧化为相应的砜, 而 BVMO4 则优先选择未取代的芳族硫化物，除此以外 也能转化在对位(甲基，氟)或间位(氯)上含有小取代基 的底物 ${ }^{[22]}$. Bornscheuer 等 ${ }^{[69]}$ 在 2017 年发现一种新的 BVMO, 即 YMOA(来源于 Yarrowia lipolytica), 它对硫 醚和亚砜都有转化能力, 甚至可以转化 DMSO. 对其进 行理性设计得到的 YMOA V121T 突变株能够增加砜产 物的形成，而 YMOA C480F 和 YMOA A483L 突变株则 只产生亚砜产物. 表明可以通过对 BVMO 的理性设计 来控制硫醚类底物在反应中的氧化程度.

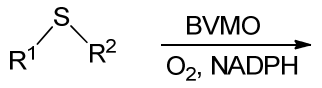<smiles>[R][Si]([CH])(O)O</smiles><smiles>CSc1ccccc1</smiles><smiles>Brc1ccccc1CSc1ccccc1</smiles>
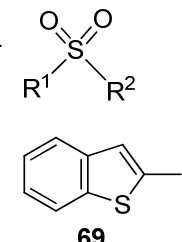

图式 21 BVMO 催化硫醚类化合物的氧化反应 Scheme 21 Heteroatom oxidation reaction of thioethers catalyzed by BVMOs

苯甲基硫醚 66 体积相对较小，一些野生型 BVMO 对这种底物本身就有很强的活性和立体选择性. 例如, $\mathrm{CHMO}_{\mathrm{Tm}}$ (来源于 Thermocrispum municipale DSM 44069 的环己酮单加氧酶)在转化这种底物时主要生成 $R$ 构型 产物 (ee 97\%) ${ }^{[70]}$, 而 $\mathrm{BVMO}_{\mathrm{Afl} 1}$ (来源于 A. fumigatus Af293)在转化这种底物时产率较高 (3h 后转化 $88 \%$ ), 只 生成 $S$ 构型亚砜 $(e e=99 \%)^{[71]}$. $\mathrm{CHMO}_{\text {Acineto }}$ 和 $\mathrm{FDH}$ (甲酸 脱氢酶)共表达时，显著提高了细胞对 NADPH 的再生能 力, 获得高立体选择性的亚砜产物(ee 99\% $)^{[72]}$. 但有些 BVMO, 如 PAMO, 对硫醚氧化的选择性并不好，一般 都得到低 $e e$ 值甚至消旋的产物 ${ }^{[22]}$, 因此也涌现出许多 通过定向进化和理性设计来改进 BVMO 对硫醚类底物 立体选择性的研究. 例如, Reetz 等 ${ }^{[73]}$ 在 2014 年将 ISM 策略用于 PAMO 的定向进化，提高其对硫醚类底物的立 体选择性. 在篎选结果中发现 PAMO I67Q/P440F/ A442N/L443I 突变株转化苯甲基硫醚 66 和其他硫醚时 能获得高立体选择性的 $R$ 构型亚砜产物 $(e e>95 \%)$. 值 得注意的是，构成这 4 倍体突变的 4 种单倍体 PAMO 突 变株都是 $S$ 构型选择性的, 而这个 4 倍体突变的转化产 物则是 $R$ 构型，再次证明了同一催化属性的突变集中后 其作用未必是叠加的. 另外以 PAMO 为骨架进行亚域交 换产生的嵌合酶 PASTMO ${ }^{[55]}$ 和 PAMEMO1 ${ }^{[55]}$, 与野生 
型 STMO 相比, 在转化苯甲基硫醚 $\mathbf{6 6}$ 时也体现出了更 高的立体选择性.

通过调整 BVMO 底物结合位点附近的氨基酸残基, 可以让改造后的酶转化原本野生型无法接受的大体积 底物, 从而扩大底物谱. 例如, PAMO M446G $\mathrm{G}^{[19]}$ 、 PAMO M446A ${ }^{[74]}$ 以及 PAMO I67S 突变株 ${ }^{[74]}$, 这三种 PAMO 突 变株通过将活性位点附近的氨基酸残基突变为体积较 小的氨基酸, 减小了空间位阻, 使其能够转化像苄基苯 基硫醚 67 这样的大体积底物, 并有着优异的立体选择 性 ${ }^{[5]}$. SMO(来源于 Pseudomonas putida CA-3 的苯乙烯 单加氧酶)对硫醚类底物本身就有较高的立体选择性, 但其对苯并 $[b]$ 噻吩 68 和 2-甲基苯并 $[b]$ 噻吩 69 这 2 种 底物的反应速率极低, 可能是因为这 2 种底物有较强的 刚性和更大的杂环结构. 而这种酶的工程酶 SMO R3-11 上的 $\beta$ 链在酶构型上有显著的变化, 改变了活性位点, 扩大了结合口袋, 其转化这 2 种底物的活性分别是野生 型 SMO 的 10.1 倍和 5.6 倍 ${ }^{[75]}$. 此外, 利用 Type I BVMO 指纹序列对微生物基因组进行基因挖掘发现的 $\mathrm{BVMO}_{\mathrm{Bo}}$ (来源于 Bradyrhizobium oligotrophicum) 和 $\mathrm{BVMO}_{\mathrm{Am}}$ (来源于 Aeromicrobium marinum) 也对多种体 积庞大的前手性硫醚(如吡唑硫醚等), 具有出色的立体 选择性和活性 ${ }^{[76]}$.

\section{3 含硒有机化合物}

受到硫化物立体选择性氧化的启发, 并考虑到硫和 硒类似的化学性质, BVMO 研究也进一步拓展到含硒有 机化合物. Brondani 等 ${ }^{[77]}$ 在 2011 年发现 PAMO 在转化 一些对位和间位含硒的苯乙酮衍生物时, 发现底物的酮 部分保留完整, 主要生成相应的硒亚砜. 次年, 他们通 过 PAMO 催化芳环中没有取代基的有机硒苯乙基化合 物 70 动力学拆分反应, 获得了 $e e$ 值高达 $98 \%$ 的 $S$ 构型 底物(Scheme 22) ${ }^{[78]}$.

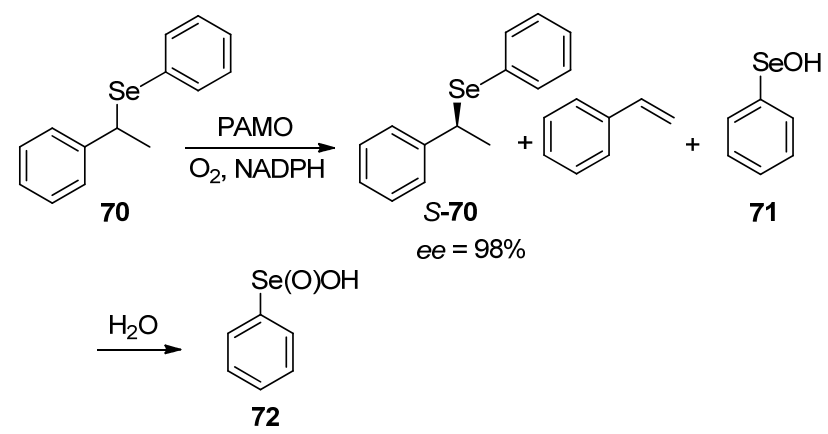

图式 22 PAMO 催化的含硒芳香化合物的动力学拆分反应 Scheme 22 Oxidative kinetic resolution of selenium-containing aromatic compounds catalyzed by BVMOs

\section{6 总结与展望}

综上所述, 随着近年来 BVMO 相关研究的不断深
入，其催化的反应类型不断得到扩展，底物结构也日渐 丰富, BVMO 的催化活性和立体选择性都得到了显著提 高. 特别是, 通过 BVMO 的特征序列从各类微生物 DNA 中快速篎选可能存在的 BVMO 序列, 并通过克隆、 重组等方法获取的新型 BVMO 不断涌现. 随着越来越 多 BVMO 的晶体结构被构建, 基于晶体结构分析以及 生物计算的理性设计已经成为研究的主要方向, 用于改 进野生型 BVMO. 虽然 BVMO 在选择性、反应条件绿 色化和催化效率等方面优于传统的化学氧化, 但要在工 业领域大范围应用还存在诸多困难. 由于 BVMO 对辅 因子的依赖性, 在有机合成中利用 BVMO 变得复杂, 特 别是在无细胞环境下利用纯酶进行反应时, $\mathrm{NAD}(\mathrm{P}) \mathrm{H}$ 的 消耗成为一项不得不考虑的成本因素, 发展新的辅酶再 生系统, 利用相对低廉的辅助底物来再生 $\mathrm{NAD}(\mathrm{P}) \mathrm{H}$ 将 是非常重要的课题; 而通过全细胞环境介导的生物转化 中, 减少反应的副产物, 寻找更廉价的诱导剂以及进一 步提高酶的活性, 也是今后探索的方向; 除此之外, 还 存在酶稳定性不足以及底物/产物抑制作用等其他问题. 因此，随着环境友好型工艺取代传统化学工艺的迫切性 不断增加, 未来如何将实验室规模的 BVMO 研究成果 放大到大规模工业生产中将是一个非常有价值的挑战.

\section{References}

[1] Balke, K.; Kadow, M.; Mallin, H.; Sass, S.; Bornscheuer, U. T. Org Biomol. Chem. 2012, 10, 6249.

[2] Leisch, H.; Morley, K.; Lau, P. C. Chem. Rev. (Washington, DC, U. S.) 2011, 111, 4165 .

[3] (a) Dong, J.; Fernandez-Fueyo, E.; Hollmann, F.; Paul, C. E.; Pesic, M.; Schmidt, S.; Wang, Y.; Younes, S.; Zhang, W. Angew. Chem., Int. Ed. 2018, 57, 9238.

(b) Balke, K.; Beier, A.; Bornscheuer, U. T. Biotechnol. Adv. 2018, 36, 247.

(c) Liang, Y.; Wei, J.; Qiu, X.; Jiao, N. Chem. Rev. 2018, 118, 4912.

[4] Ceccoli, R. D.; Bianchi, D. A.; Fink, M. J.; Mihovilovic, M. D.; Rial, D. V. $A M B$ Express 2017, 7, 87.

[5] Beneventi, E.; Niero, M.; Motterle, R.; Fraaije, M.; Bergantino, E. J. Mol. Catal. B: Enzym. 2013, 98, 145.

[6] Fiorentini, F.; Romero, E.; Fraaije, M. W.; Faber, K.; Hall, M.; Mattevi, A. ACS Chem Biol. 2017, 12, 2379.

[7] Fink, M. J.; Mihovilovic, M. D. Chem. Commun. (Cambridge, U. K.) 2015, 51, 2874.

[8] van Beek, H. L.; Romero, E.; Fraaije, M. W. ACS Chem Biol. 2017, $12,291$.

[9] Pereira, J. P. C.; van der Wielen, L. A. M.; Straathof, A. J. J. Bioresour. Technol. 2018, 256, 187.

[10] Carvalho, A. T. P.; Dourado, D.; Skvortsov, T.; de Abreu, M.; Ferguson, L. J.; Quinn, D. J.; Moody, T. S.; Huang, M. Phys. Chem. Chem. Phys. 2018, 20, 2558.

[11] Song, J. W.; Jeon, E. Y.; Song, D. H.; Jang, H. Y.; Bornscheuer, U. T.; Oh, D. K.; Park, J. B. Angew. Chem., Int. Ed. 2013, 52, 2534.

[12] Jeon, E.-Y.; Seo, J.-H.; Kang, W.-R.; Kim, M.-J.; Lee, J.-H.; Oh, D.-K.; Park, J.-B. ACS Catal. 2016, 6, 7547.

[13] Seo, E. J.; Yeon, Y. J.; Seo, J. H.; Lee, J. H.; Bongol, J. P.; Oh, Y.; Park, J. M.; Lim, S. M.; Lee, C. G.; Park, J. B. Bioresour. Technol. 2018, 251, 288.

[14] (a) Rehdorf, J.; Zimmer, C. L.; Bornscheuer, U. T. Appl. Environ. Microbiol. 2009, 75, 3106. 
(b) Geitner, K.; Rehdorf, J.; Snajdrova, R.; Bornscheuer, U. T. Appl. Microbiol. Biotechnol. 2010, 88, 1087.

[15] Riebel, A.; Dudek, H. M.; de Gonzalo, G.; Stepniak, P.; Rychlewski, L.; Fraaije, M. W. Appl. Microbiol. Biotechnol. 2012, 95, 1479.

[16] Ferroni, F. M.; Smit, M. S.; Opperman, D. J. J. Mol. Catal. B: Enzym. 2014, 107, 47

[17] (a) Fraaije, M. W.; Wu, J.; Heuts, D. P.; van Hellemond, E. W.; Spelberg, J. H.; Janssen, D. B. Appl. Microbiol. Biotechnol. 2005, $66,393$.

(b) de Gonzalo, G.; Mihovilovic, M. D.; Fraaije, M. W. ChemBioChem 2010, 11, 2208.

[18] Pazmino, D. E. T.; Snajdrova, R.; Rial, D. V.; Mihovilovic, M. D.; Fraaije, M. W. Adv. Synth. Catal. 2007, 349, 1361.

[19] Dudek, H. M.; de Gonzalo, G.; Pazmino, D. E.; Stepniak, P.; Wyrwicz, L. S.; Rychlewski, L.; Fraaije, M. W. Appl. Environ. Microbiol. 2011, 77, 5730 .

[20] Dudek, H. M.; Fink, M. J.; Shivange, A. V.; Dennig, A.; Mihovilovic, M. D.; Schwaneberg, U.; Fraaije, M. W. Appl. Microbiol. Biotechnol. 2014, 98, 4009.

[21] Franceschini, S.; van Beek, H. L.; Pennetta, A.; Martinoli, C.; Fraaije, M. W.; Mattevi, A. J. Biol. Chem. 2012, 287, 22626.

[22] Bisagni, S.; Summers, B.; Kara, S.; Hatti-Kaul, R.; Grogan, G.; Mamo, G.; Hollmann, F. Top. Catal. 2013, 57, 366.

[23] Messiha, H. L.; Ahmed, S. T.; Karuppiah, V.; Suardiaz, R.; Ascue Avalos, G. A.; Fey, N.; Yeates, S.; Toogood, H. S.; Mulholland, A. J.; Scrutton, N. S. Biochemistry 2018, 57, 1997.

[24] Alexander, A. K.; Biedermann, D.; Fink, M. J.; Mihovilovic, M. D.; Mattes, T. E. J. Mol. Catal. B: Enzym. 2012, 78, 105.

[25] Fink, M. J.; Fischer, T. C.; Rudroff, F.; Dudek, H.; Fraaije, M. W.; Mihovilovic, M. D. J. Mol. Catal. B: Enzym. 2011, 73, 9.

[26] Rudroff, F.; Fink, M. J.; Pydi, R.; Bornscheuer, U. T.; Mihovilovic, M. D. Monatsh. Chem. 2017, 148, 157.

[27] Balke, K.; Schmidt, S.; Genz, M.; Bornscheuer, U. T. ACS Chem Biol. 2016, 11, 38 .

[28] Zhang, Z. G.; Parra, L. P.; Reetz, M. T. Chem.-Eur. J. 2012, 18, 10160.

[29] Rodríguez-Mata, M.; Lavandera, I.; Gotor-Fernández, V.; Gotor, V.; García-Cerrada, S.; Mendiola, J.; de Frutos, Ó.; Collado, I. Tetrahedron 2016, 72, 7268 .

[30] Reetz, M. T.; Brunner, B.; Schneider, T.; Schulz, F.; Clouthier, C. M.; Kayser, M. M. Angew. Chem., Int. Ed. 2004, 43, 4075.

[31] Clouthier, C. M.; Kayser, M. M.; Reetz, M. T. J. Org. Chem. 2006, 71,8431 .

[32] Polyak, I.; Reetz, M. T.; Thiel, W. J. Phys. Chem. B 2013, 117, 4993.

[33] Zhang, Z.-G.; Roiban, G.-D.; Acevedo, J. P.; Polyak, I.; Reetz, M. T. Adv. Synth. Catal. 2013, 355, 99.

[34] Parra, L. P.; Agudo, R.; Reetz, M. T. ChemBioChem 2013, 14, 2301.

[35] Wu, S.; Acevedo, J. P.; Reetz, M. T. Proc. Natl. Acad. Sci. U. S. A. 2010, 107, 2775.

[36] Yachnin, B. J.; McEvoy, M. B.; MacCuish, R. J.; Morley, K. L.; Lau, P. C.; Berghuis, A. M. ACS Chem Biol. 2014, 9, 2843.

[37] Iwaki, H.; Grosse, S.; Bergeron, H.; Leisch, H.; Morley, K.; Hasegawa, Y.; Lau, P. C. Appl. Environ. Microbiol. 2013, 79, 3282.

[38] Schmidt, S.; Genz, M.; Balke, K.; Bornscheuer, U. T. J. Biotechnol. 2015, 214, 199.

[39] Mallin, H.; Wulf, H.; Bornscheuer, U. T. Enzyme Microb. Technol. 2013, 53, 283.

[40] Staudt, S.; Bornscheuer, U. T.; Menyes, U.; Hummel, W.; Groger, H. Enzyme Microb. Technol. 2013, 53, 288

[41] Oberleitner, N.; Peters, C.; Rudroff, F.; Bornscheuer, U. T.; Mihovilovic, M. D. J. Biotechnol. 2014, 192, 393.

[42] Schmidt, S.; Scherkus, C.; Muschiol, J.; Menyes, U.; Winkler, T.; Hummel, W.; Groger, H.; Liese, A.; Herz, H. G.; Bornscheuer, U. T. Angew. Chem., Int. Ed. 2015, 54, 2784.

[43] Milker, S.; Fink, M. J.; Oberleitner, N.; Ressmann, A. K.;
Bornscheuer, U. T.; Mihovilovic, M. D.; Rudroff, F. ChemCatChem 2017, 9, 3420

[44] Kohl, A.; Srinivasamurthy, V.; Bottcher, D.; Kabisch, J.; Bornscheuer, U. T. Enzyme Microb. Technol. 2018, 108, 53.

[45] Reignier, T.; de Berardinis, V.; Petit, J. L.; Mariage, A.; Hamze, K.; Duquesne, K.; Alphand, V. Chem. Commun. (Cambridge, U. K.) 2014, 50, 7793.

[46] Morrill, C.; Jensen, C.; Just-Baringo, X.; Grogan, G.; Turner, N. J.; Procter, D. J. Angew. Chem., Int. Ed. 2018, 57, 3692.

[47] Kadow, M.; Loschinski, K.; Sass, S.; Schmidt, M.; Bornscheuer, U. T. Appl. Microbiol. Biotechnol. 2012, 96, 419.

[48] Fink, M. J.; Rial, D. V.; Kapitanova, P.; Lengar, A.; Rehdorf, J.; Cheng, Q.; Rudroff, F.; Mihovilovic, M. D. Adv. Synth. Catal. 2012, 354,3491 .

[49] Leipold, F.; Wardenga, R.; Bornscheuer, U. T. Appl. Microbiol. Biotechnol. 2012, 94, 705.

[50] Kadow, M.; Balke, K.; Willetts, A.; Bornscheuer, U. T.; Backvall, J. E. Appl. Microbiol. Biotechnol. 2014, 98, 3975.

[51] Furst, M. J.; Savino, S.; Dudek, H. M.; Gomez Castellanos, J. R.; Gutierrez de Souza, C.; Rovida, S.; Fraaije, M. W.; Mattevi, A. J. Am. Chem. Soc. 2017, 139, 627.

[52] Balke, K.; Baumgen, M.; Bornscheuer, U. T. ChemBioChem 2017, $18,1627$.

[53] Butinar, L.; Mohorcic, M.; Deyris, V.; Duquesne, K.; Iacazio, G.; Claeys-Bruno, M.; Friedrich, J.; Alphand, V. Phytochemistry 2015, $117,144$.

[54] Romero, E.; Castellanos, J. R.; Mattevi, A.; Fraaije, M. W. Angew. Chem., Int. Ed. 2016, 55, 15852.

[55] van Beek, H. L.; de Gonzalo, G.; Fraaije, M. W. Chem. Commun (Cambridge, U. K.) 2012, 48, 3288

[56] Mascotti, M. L.; Palazzolo, M. A.; Bisogno, F. R.; Kurina-Sanz, M. Steroids 2016, 109, 44.

[57] Bosserman, M. A.; Downey, T.; Noinaj, N.; Buchanan, S. K.; Rohr, J. ACS Chem Biol. 2013, 8, 2466 .

[58] Chen, K.; Wu, S.; Zhu, L.; Zhang, C.; Xiang, W.; Deng, Z.; Ikeda, H.; Cane, D. E.; Zhu, D. Biochemistry 2016, 55, 6696.

[59] Minerdi, D.; Zgrablic, I.; Castrignano, S.; Catucci, G.; Medana, C.; Terlizzi, M. E.; Gribaudo, G.; Gilardi, G.; Sadeghi, S. J. Antimicrob. Agents Chemother. 2016, 60, 64

[60] Qiao, K.; Chooi, Y. H.; Tang, Y. Metab. Eng. 2011, 13, 723.

[61] Hu, Y.; Dietrich, D.; Xu, W.; Patel, A.; Thuss, J. A.; Wang, J.; Yin, W. B.; Qiao, K.; Houk, K. N.; Vederas, J. C.; Tang, Y. Nat. Chem. Biol. 2014, 10, 552.

[62] de Gonzalo, G.; Torres Pazmiño, D. E.; Ottolina, G.; Fraaije, M. W.; Carrea, G. Tetrahedron: Asymmetry 2006, 17, 130.

[63] (a) Branchaud, B. P.; Walsh, C. T. J. Am. Chem. Soc. 1985, 107 2153.

(b) Walsh, C. T.; Chen, Y. C. J. Angew. Chem., Int. Ed. Engl. 1988, 27,333 .

[64] Gonzalo, G. D.; Pazmiño, D. E. T.; Ottolina, G.; Fraaije, M. W.; Carrea, G. Tetrahedron: Asymmetry 2005, 16, 3077.

[65] Ceccoli, R. D.; Bianchi, D. A.; Rial, D. V. Front. Microbiol. 2014, 5,25 .

[66] Brondani, P. B.; de Gonzalo, G.; Fraaije, M. W.; Andrade, L. H. Adv. Synth. Catal. 2011, 353, 2169.

[67] Das, B. C.; Thapa, P.; Karki, R.; Schinke, C.; Das, S.; Kambhampati, S.; Banerjee, S. K.; Van Veldhuizen, P.; Verma, A.; Weiss, L. M.; Evans, T. Future Med. Chem. 2013, 5, 653.

[68] Alphand, V.; Wohlgemuth, R. Curr. Org. Chem. 2010, 14, 1928.

[69] Bordewick, S.; Beier, A.; Balke, K.; Bornscheuer, U. T. Enzyme Microb. Technol. 2018, 109, 31.

[70] de Gonzalo, G.; Franconetti, A. Enzyme Microb. Technol. 2018, 113, 24.

[71] Mascotti, M. L.; Juri Ayub, M.; Dudek, H.; Sanz, M. K.; Fraaije, M. W. AMB Express 2013, 3, 33.

[72] Zhai, X. H.; Ma, Y. H.; Lai, D. Y.; Zhou, S.; Chen, Z. M. J. Ind. Microbiol. Biotechnol. 2013, 40, 797.

[73] Zhang, Z. G.; Lonsdale, R.; Sanchis, J.; Reetz, M. T. J. Am. Chem. 
Soc. 2014, 136, 17262.

[74] Dudek, H. M.; Popken, P.; van Bloois, E.; Duetz, W. A.; Fraaije, M. W. J. Biomol. Screening. 2013, 18, 678.

[75] Nikodinovic-Runic, J.; Coulombel, L.; Francuski, D.; Sharma, N. D.; Boyd, D. R.; Ferrall, R. M.; O'Connor, K. E. Appl. Microbiol. Biotechnol. 2013, 97, 4849.
[76] Zhang, Y.; Liu, F.; Xu, N.; Wu, Y. Q.; Zheng, Y. C.; Zhao, Q.; Lin, G.; Yu, H. L.; Xu, J. H. Appl. Environ. Microbiol. 2018, 84.

[77] Andrade, L. H.; Pedrozo, E. C.; Leite, H. G.; Brondani, P. B. J. Mol. Catal. B: Enzym. 2011, 73, 63.

[78] Brondani, P. B.; Guilmoto, N. M. A. F.; Dudek, H. M.; Fraaije, M. W.; Andrade, L. H. Tetrahedron 2012, 68, 10431.

(Lu, Y.) 